\title{
On a paper by Copson and Ferrar
}

\author{
By A. Erdélyt. \\ [Extract from a letter to W. L. Ferrar.]
}

Concerning your paper ${ }^{1}$ written in collaboration with Professor Copson, I found the expansion of

$$
F(\lambda)=\frac{1}{2 \pi} \int_{0}^{\infty} e^{i \lambda \cosh t} \frac{\sin \theta}{\cosh t+\cos \theta} d t
$$

for small values of $\lambda$ in the following manner: The elementary substitution $\tan \frac{1}{2} \phi=\sin \theta /\left(e^{t}+\cos \theta\right)$ yields

$$
F(0)=\frac{1}{2 \pi} \int_{0}^{\infty} \frac{\sin \theta}{\cosh t+\cos \theta} d t=\frac{1}{2 \pi} \int_{0}^{\theta} d \phi=\frac{\theta}{2 \pi},
$$

i.e. your equation (5.11). By reason of the differential equation

$$
\frac{d F}{d \lambda}+i \cos \theta \cdot F=-\frac{1}{4} \sin \theta \cdot H_{0}^{(1)}(\lambda), \quad F^{\prime}(0)=\frac{\theta}{2 \pi},
$$

it follows that

$$
F(\lambda)=e^{-i \lambda \cos \theta}\left\{\frac{\theta}{2 \pi}-\frac{1}{4} \sin \theta \cdot \int_{0}^{\lambda} H_{0}^{(1)}(\nu) e^{i \nu \cos \theta} d \nu\right\} .
$$

Thus I have expressed $F(\lambda)$ as a finite integral. Expansion of the integrand yields at once the expansion of $F(\lambda)$ for small values of $\lambda$.

1 Proc. Edinburgh Math. Soc. (2), 5 (1938), 159-168.

BRUenN, May 5th, 1938. 